\title{
Digestive System Perforation
}

National Cancer Institute

\section{Source}

National Cancer Institute. Digestive System Perforation. NCI Thesaurus. Code C79470.

A rupture in the wall of any structure of the digestive system caused by traumatic or pathologic processes. 